\title{
Nanoparticle manipulation through inter-particle optical forces and torques
}

\author{
David S. Bradshaw and David L. Andrews* \\ Nanostructures and Photomolecular Systems, School of Chemical Sciences \\ University of East Anglia, Norwich NR4 7TJ, U.K.
}

\begin{abstract}
Recently, emerging from studies based on quantum electrodynamics, it has been shown possible to significantly modify the form and magnitude of inter-particle forces by the throughput of intense laser light. This paper identifies these laser-induced forces as being the result of coherent stimulated scattering by particle pairs. Such forces have the capacity to generate novel patterns of nanoscale response, entirely determined and controlled by the frequency, intensity, polarisation and other features of the laser input. Results are given, based on general calculations of the optical forces and torques operating between a pair of dielectric particles. It is subsequently shown, by further development of the analysis, that it is possible to address the case of a twisted (Laguerre-Gaussian) laser beam as the input radiation. Here, the results reveal additional and highly distinctive torques operating between pairs of nanoparticles. Significantly the results demonstrate that these laser-induced forces and torques can be either positive or negative according to conditions. As a consequence, new possibilities emerge for the optical control of nanoparticle ordering, clustering and trapping.
\end{abstract}

Keywords: Optical forces, optical vortices, twisted beams, Laguerre-Gaussian beams, quantum electrodynamics, nanoparticles, carbon nanotubes, optical tweezers, stimulated scattering

\section{INTRODUCTION}

During the early years of laser development the practical utilization of optomechanical forces to manipulate small particles was identified in pioneering work of Ashkin. ${ }^{1}$ The new field evolved rapidly and eventually led to the

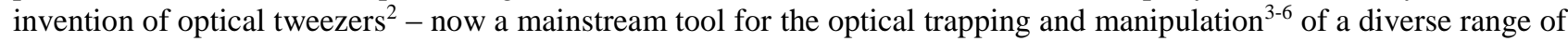
particles. More recently, by employing 'twisted' Laguerre-Gaussian (LG) input beams, optical tweezers have been developed that not only trap particles but also rotate them - these techniques have become known as 'optical spanners' and result from angular momentum transfer. ${ }^{7,8}$ Optical trapping and manipulation are generally based on the attraction of particles towards the high intensity region of a laser beam. In systems comprising two or more particles, subjected to these optomechanical forces, it is clear that static, inter-particle forces are also present. The fundamental character of such forces is very well known and, for particles separated beyond the region of wavefunction overlap, derives from dipole interactions, dispersion forces etc. Less well known, but operating alongside these static interactions when laser radiation is present, are radiation-induced inter-particle forces. The concept of these laser-induced coupling forces was first identified by Thirunamachandran, ${ }^{9}$ in a treatment based on quantum electrodynamics (QED) - a theory that designedly addresses the quantum interactions of matter with radiation. In extension to this work, ${ }^{10}$ it has more recently emerged that these laser-induced forces can variously modify the form and magnitude of inter-particle coupling forces. Moreover, these effects are particularly significant in the case of nanoscale particles. Thus, entirely distinct and separable from the optomechanical interactions involved in optical tweezers, laser-induced inter-particle forces and torques are capable of generating novel patterns of nanoparticle motion, ultimately determined by the intensity, polarization and other features of the laser input. In the following section of this paper a general expression is presented for the laser-induced force between dielectric particles. By employing results established for the interaction of LG or other 'twisted' optical beams with matter, ${ }^{11}$ the following section outlines a general expression for the torque between a pair of particles in an LG beam. Finally, for irradiating LG light, novel features are exemplified by the first application, a system of spherical nanoparticles. 


\section{LASER-INDUCED FORCE EXPRESSION}

In order to properly represent the quantum features of the radiation we shall develop the theory using the framework of quantum electrodynamics. ${ }^{12}$ This framework - in which both radiation and matter are treated quantum mechanically provides a highly rigorous and satisfactory theory for the study of optically-induced particle interactions. Using the Power-Zienau-Woolley approach, ${ }^{13}$ the full multipolar Hamiltonian, $H$, for a pair of particles A and B is given by;

$$
H=\sum_{\xi=A, B} H_{\mathrm{mol}}(\xi)+\sum_{\xi=A, B} H_{\mathrm{int}}(\xi)+H_{\mathrm{rad}}
$$

where $H_{\text {mol }}^{\xi}$ is the multipolar Hamiltonian for particle $\xi$ and $H_{\text {rad }}$ denotes the radiation field. The Hamiltonian $H_{\text {int }}^{\xi}$ represents the interaction of the field with $\xi$ and, within the electric dipole, is given by;

$$
H_{\mathrm{int}}^{\xi}=-\varepsilon_{0}^{-1} \sum_{\xi} \boldsymbol{\mu}(\xi) \cdot \mathbf{d}^{\perp}\left(\mathbf{R}_{\xi}\right)
$$

with $\mu(\xi)$ and $\mathbf{R}_{\xi}$ as the electric-dipole moment operator and the position vector, respectively. The operator $\mathbf{d}^{\perp}\left(\mathbf{R}_{\xi}\right)$ represents the transverse electric displacement field which is generally (but not necessarily) quantised within a volume $V$ and cast as a plane wave mode expansion involving summations over all wave-vectors, $\mathbf{p}$, and polarisations, $\mathbf{e}^{(\lambda)}(\mathbf{p})$. The latter unit vector is taken as complex to accommodate circular or elliptical polarisations, and its complex conjugate is written as $\overline{\mathbf{e}}^{(\lambda)}(\mathbf{p})$. A general result for a laser-induced force between a pair of neutral species A and B can be determined from an expression for the distance-dependent energy shift, $\Delta E_{\text {ind }}$, which in turn is obtained by the application of fourth-order perturbation theory;

$$
\Delta E_{\text {ind }}=\operatorname{Re}\left\{\sum_{t, s, r} \frac{\left\langle i\left|H_{\mathrm{int}}\right| t\right\rangle\left\langle t\left|H_{\mathrm{int}}\right| s\right\rangle\left\langle s\left|H_{\mathrm{int}}\right| r\right\rangle\left\langle r\left|H_{\mathrm{int}}\right| i\right\rangle}{\left(E_{i}-E_{t}\right)\left(E_{i}-E_{s}\right)\left(E_{i}-E_{r}\right)}\right\},
$$

where $|i\rangle$ is the unperturbed state in which both A and B are in their electronic ground state; $|r\rangle,|s\rangle$ and $|t\rangle$ are virtual states, and $E_{n}$ is the energy of state $|n\rangle$. These states can be written in the form;

$$
|n\rangle=\left|\operatorname{mol}_{n}\right\rangle\left|\operatorname{rad}_{n}\right\rangle \equiv\left|\operatorname{mol}_{n} ; \operatorname{rad}_{n}\right\rangle
$$

with $\left|\operatorname{mol}_{n}\right\rangle$ and $\left|\operatorname{rad}_{n}\right\rangle$ defining the status of all particle and radiation states, respectively. The laser-induced interaction entails two distinct mechanisms. One involves the absorption of a 'real' (laser throughput) photon at one molecule and the stimulated emission of a 'real' photon at the other, with a virtual photon acting as mediator between them. Various quantum channels are involved in this mechanism, their totality concisely depicted by the state-sequence diagram shown in fig. 1 (for details, see the Appendix). This is a dynamic form of coupling, so-called because a real and finite amount of energy is conveyed between the two particles. The second mechanism for laser-induced inter-particle interaction entails real photon creation and annihilation events occurring at one molecule, with coupling to the other by a virtual mediator (fig. 2); this represents a static form of coupling. The particles and the throughput radiation suffer no overall change in quantum state. As shown previously (using Feynman diagram methods) ${ }^{14}$ the result for $\Delta E_{\text {ind }}$ emerges as;

$$
\begin{aligned}
\Delta E_{\text {ind }}= & \left(\frac{n \hbar c k}{\varepsilon_{0} V}\right) \operatorname{Re}\left\{e_{i}^{(1)} \bar{e}_{l}^{(2)} \alpha_{i j}^{\mathrm{A}}(k) V_{j k}(k, \mathbf{R}) \alpha_{k l}^{\mathrm{B}}(k) \exp (i \mathbf{k} \cdot \mathbf{R})\right. \\
& \left.+\frac{1}{2} e_{i}^{(1)} \bar{e}_{l}^{(2)} V_{j k}(0, \mathbf{R})\left(\beta_{i j l}^{\mathrm{A}}(k) \mu_{k}^{\mathrm{B}}+\mu_{k}^{\mathrm{A}} \beta_{i j l}^{\mathrm{B}}(k)\right)\right\}
\end{aligned}
$$




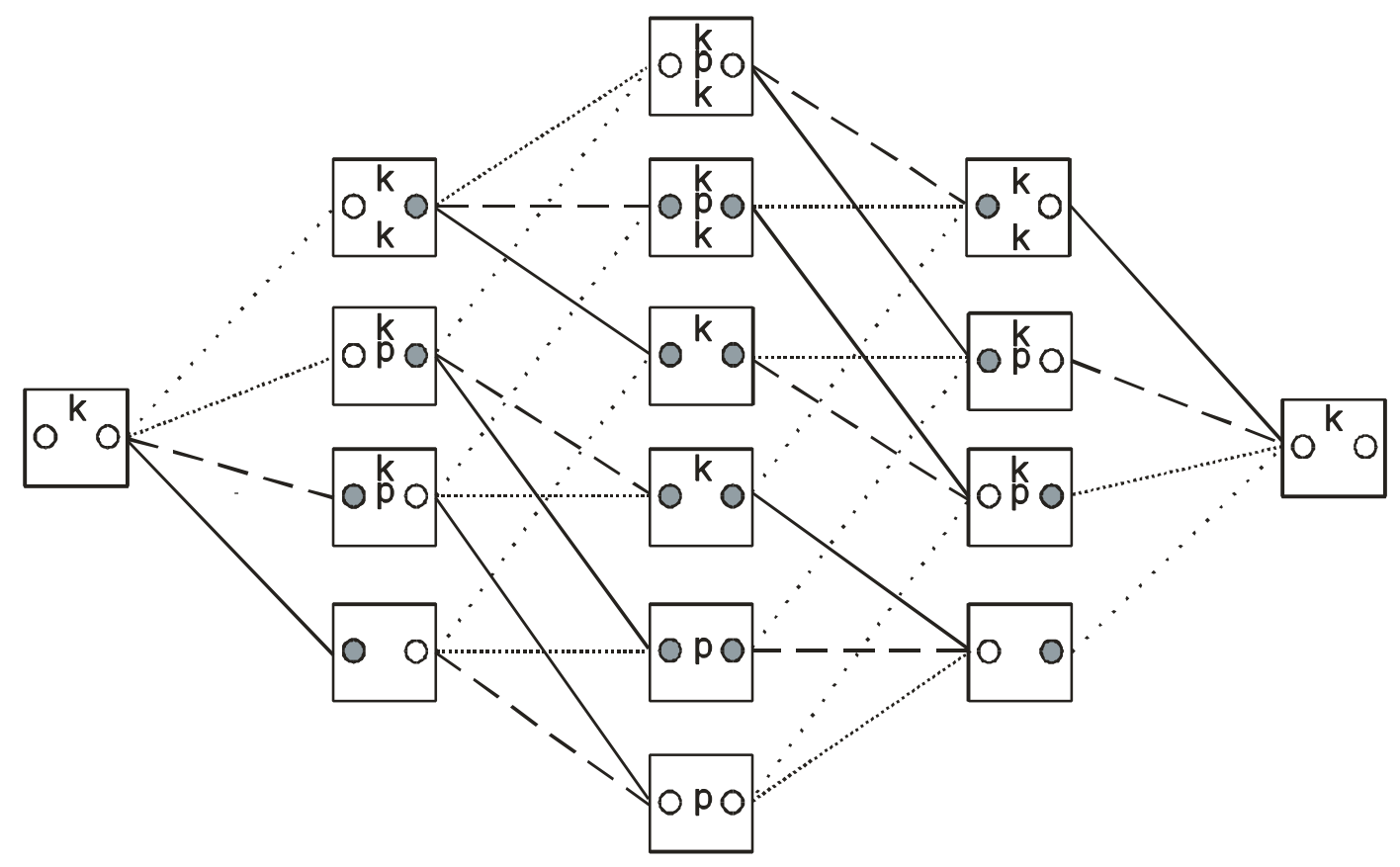

Fig. 1. State-sequence diagram for calculation of dynamic contributions to the laser-induced pair interaction, associated with the annihilation of an input photon at $\mathrm{A}$ and creation of an output photon at B. Each of the 24 paths across the diagram from left to right corresponds to a distinct Feynman graph. Key: k - 'real' (laser throughput) photon, $\mathrm{p}$ - 'virtual' photon, $\mathrm{O}$ - particle in ground state (particle $\mathrm{A}$ is a circle on the left-hand side of the box and particle B is on the right), $\mathrm{O}$-particle in virtual excited state,

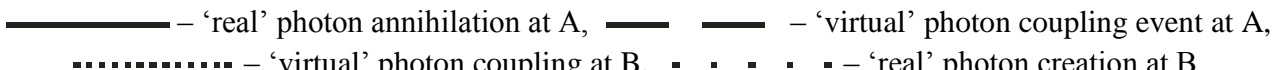

using the implied summation convention for repeated Cartesian tensor indices and assuming the dynamic polarisabilities are index-symmetric. Also in equation (5), $\mathbf{k}$ denotes the input wave-vector and $\hbar c k$ the corresponding photon energy, $V_{j k}$ signifies the fully retarded resonance electric dipole - electric dipole interaction tensor ${ }^{15}$ (dependent on the intermolecular displacement vector, $\mathbf{R} \equiv \mathbf{R}_{B}-\mathbf{R}_{A}$ ), and lastly $\mu_{k}^{\xi}, \alpha_{i j}$ and $\beta_{i j k}$ are the static (permanent) dipole moment, generalised dynamic molecular polarisability and generalised dynamic hyperpolarisability, respectively. From (5) the laser-induced force $\mathbf{F}_{\text {ind }}=-\partial \Delta E_{\text {ind }} / \partial \mathbf{R}$ emerges as follows;

$$
\begin{aligned}
F_{m}= & \left(\frac{I}{8 \pi \varepsilon_{0}^{2} c R^{4}}\right)\left[2 \operatorname { R e } ( e _ { i } ^ { ( 1 ) } \overline { e } _ { l } ^ { ( 2 ) } \alpha _ { i j } ^ { \mathrm { A } } ( k ) \alpha _ { k l } ^ { \mathrm { B } } ( k ) ) \left\{-k^{3} R^{3}\left(\cos k R \sin (\mathbf{k} \cdot \mathbf{R}) \hat{k}_{m}+\sin k R \cos (\mathbf{k} \cdot \mathbf{R}) \hat{R}_{m}\right)\right.\right. \\
& \times\left(\delta_{j k}-\hat{R}_{j} \hat{R}_{k}\right)-k^{2} R^{2}\left[\cos k R \cos (\mathbf{k} \cdot \mathbf{R})\left(2 \delta_{j k} \hat{R}_{m}+\delta_{m j} \hat{R}_{k}+\delta_{m k} \hat{R}_{j}-6 \hat{R}_{j} \hat{R}_{k} \hat{R}_{m}\right)-\hat{k}_{m} \sin k R\right. \\
& \left.\times \sin (\mathbf{k} \cdot \mathbf{R})\left(\delta_{j k}-3 \hat{R}_{j} \hat{R}_{k}\right)\right]+k R\left[3 \sin k R \cos (\mathbf{k} \cdot \mathbf{R})\left(\delta_{j k} \hat{R}_{m}+\delta_{m j} \hat{R}_{k}+\delta_{m k} \hat{R}_{j}-5 \hat{R}_{j} \hat{R}_{k} \hat{R}_{m}\right)+\hat{k}_{m}\right. \\
& \left.\left.\times \cos k R \sin (\mathbf{k} \cdot \mathbf{R})\left(\delta_{j k}-3 \hat{R}_{j} \hat{R}_{k}\right)\right]+3 \cos k R \cos (\mathbf{k} \cdot \mathbf{R})\left(\delta_{j k} \hat{R}_{m}+\delta_{m j} \hat{R}_{k}+\delta_{m k} \hat{R}_{j}-5 \hat{R}_{j} \hat{R}_{k} \hat{R}_{m}\right)\right\} \\
& \left.+3 \operatorname{Re}\left(e_{i}^{(1)} \bar{e}_{l}^{(2)}\left\{\beta_{i j l}^{\mathrm{A}}(k) \mu_{k}^{\mathrm{B}}(k)+\beta_{i j l}^{\mathrm{B}}(k) \mu_{k}^{\mathrm{A}}(k)\right\}\right)\left(\delta_{j k} \hat{R}_{m}+\delta_{m j} \hat{R}_{k}+\delta_{m k} \hat{R}_{j}-5 \hat{R}_{j} \hat{R}_{k} \hat{R}_{m}\right)\right],
\end{aligned}
$$

where $I=n \hbar c^{2} k / V$ is the input irradiance. A notable departure from an earlier, classical result ${ }^{16}$ is the inclusion in equation (6), of terms associated with the static coupling mechanism, as well as the dynamic terms. This full QED expression is more general, in the following respects: (i) the polarisability is allowed to be anisotropic and dynamic, in the sense that it has implicit frequency-dependence; (ii) the input wave-vector can have arbitrary orientations with respect to the inter-particle displacement vector, and (iii) the input radiation is also polarized arbitrarily. 


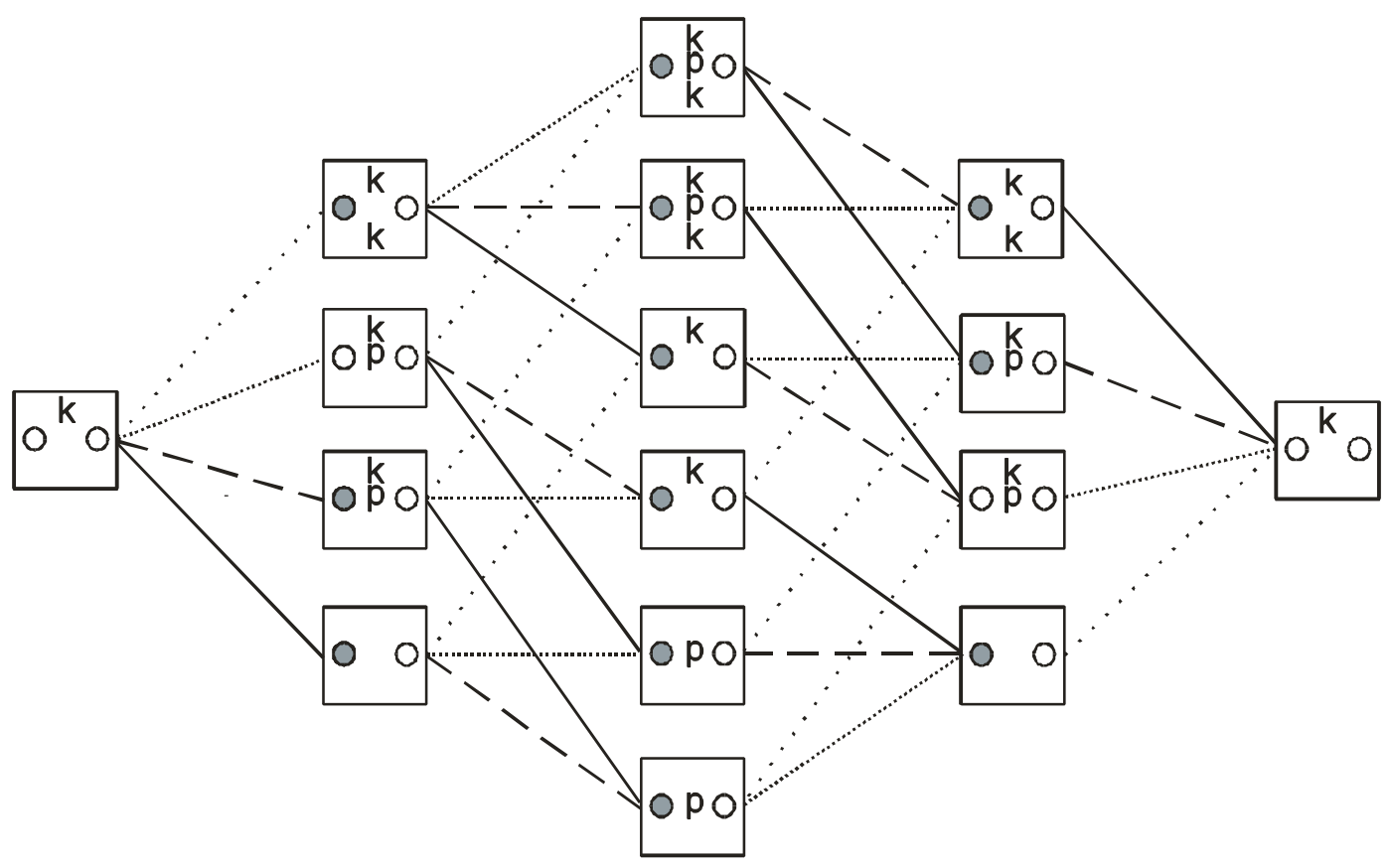

Fig. 2. State-sequence diagram for calculation of static contributions to the laser-induced interaction, associated with both the annihilation of an input photon and the creation of an output photon at A. For key, see caption to fig. 1, but here with - . . - 'real' photon creation at A.

\section{PARTICLES IN AN OPTICAL VORTEX}

\section{A. General torque expression}

Recently, optically engineered 'twisted' laser beams (optical vortices) - i.e. beams endowed with orbital angular momentum ${ }^{17,18}$ - have become the subject of considerable interest. The most widely studied case comprises LaguerreGaussian (LG) modes characterized by two integers, $l$ and $p$. Here the former number, $l$, is known as the winding number or topological charge and signifies that each photon carries orbital angular momentum of magnitude $l \hbar$, while $(p+1)$ denotes the number of radial nodes. With the appropriate quantum field representation now established, ${ }^{11}$ it is possible to formulate the involvement of twisted beams in laser-induced inter-particle forces, and to identify some distinctive features that emerge.

Fig. 3 illustrates a pair of nanoparticles (A and B) optically trapped in an LG beam with $p=0$, i.e. an optical vortex with one radial node at the beam centre. Both particles are equidistant from the beam centre and contained within the same cross-section of the beam, i.e. they are a distance, $r$, from the beam centre and confined to a particular $\xi$ value. Under these conditions, it is interesting to determine an expression for the laser-induced torque between the particles with respect to the beam axis $\xi$ - this can be achieved by first determining $\Delta E_{\text {ind }}$. Allowing the azimuthal displacement angle, $\Delta \psi=\psi_{B}-\psi_{A}$, to be the only variable and following the same procedure as section 2; applying the fourth-order perturbation method gives;

$$
\begin{aligned}
\Delta E_{\text {ind }} & =\left(\frac{I f_{l p}^{2}(r)}{\varepsilon_{0} c A_{l p}}\right) \operatorname{Re}\left\{\varepsilon_{i}^{(1)} \bar{\varepsilon}_{l}^{(2)} \alpha_{i j}^{\mathrm{A}}(k) V_{j k}(k, \mathbf{R}) \alpha_{k l}^{\mathrm{B}}(k) \exp (l \Delta \psi)\right. \\
& \left.+\frac{1}{2} \varepsilon_{i}^{(1)} \bar{\varepsilon}_{l}^{(2)} V_{j k}(0, \mathbf{R})\left(\beta_{i j l}^{\mathrm{A}}(k) \mu_{k}^{\mathrm{B}}+\mu_{k}^{\mathrm{A}} \beta_{i j l}^{\mathrm{B}}(k)\right)\right\} .
\end{aligned}
$$




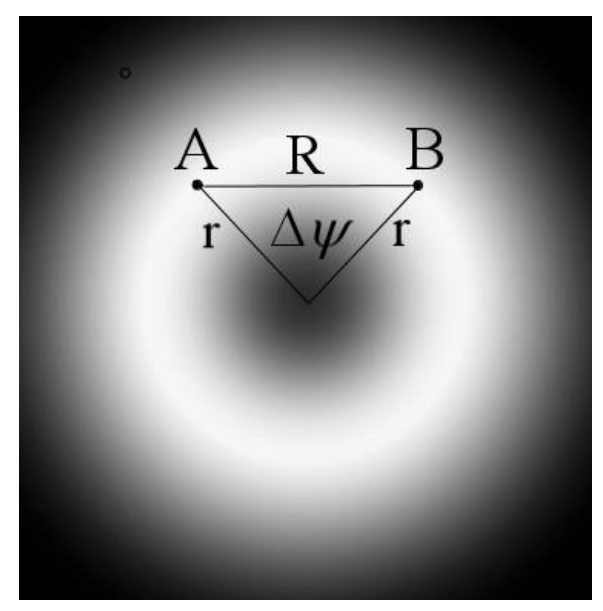

Fig. 3. Geometry of nanoparticle pair in a Laguerre-Gaussian beam.

Here, $p$ accounts for all possible modes with the same angular momentum quantum number $l, \mathrm{~A}_{l p}$ are normalisation constants, and $f_{l p}(r)$ is given by;

$$
f_{l p}(r)=\frac{C_{p}^{|l|}}{\omega_{0}}\left[\frac{\sqrt{2} r}{\omega_{0}}\right]^{|l|} \exp \left(\frac{-r^{2}}{\omega_{0}^{2}}\right) L_{p}^{|l|}\left(\frac{2 r^{2}}{\omega_{0}^{2}}\right),
$$

where $C_{p}^{|l|}$ is a normalisation constant, $\omega_{0}$ defines the Gaussian beam-waist at $\mathrm{z}=0$ and $L_{p}^{|l|}$ is the generalised Laguerre polynomial. Using $R=\sqrt{2} r(1-\cos \Delta \psi)^{1 / 2}$, the laser-induced torque $\tau=\left(-\partial \Delta E_{\text {ind }} / \partial \Delta \varphi\right) \hat{\mathbf{R}}$ now emerges as;

$$
\begin{aligned}
\tau & =\left(\frac{I k^{3} f_{l p}^{2} \hat{\mathbf{R}}}{8 \pi \varepsilon_{0}^{2} c A_{l p}}\right)\left[2 \operatorname { R e } ( \varepsilon _ { i } ^ { ( 1 ) } \overline { \varepsilon } _ { l } ^ { ( 2 ) } \alpha _ { i j } ^ { A } \alpha _ { k l } ^ { B } ) \left\{-\frac{\left(\delta_{j k}-\hat{R}_{j} \hat{R}_{k}\right) \sin \left(\sqrt{2} k r(1-\cos \Delta \psi)^{1 / 2}\right) \sin \Delta \psi}{2(1-\cos \Delta \psi)}\right.\right. \\
& \times \cos (l \Delta \psi)-\frac{\cos \left(\sqrt{2} k r(1-\cos \Delta \psi)^{1 / 2}\right)}{\sqrt{2} k r(1-\cos \Delta \psi)^{3 / 2}}\left(\left(\delta_{j k}-\hat{R}_{j} \hat{R}_{k}\right) l \sin (l \Delta \psi)(1-\cos \Delta \psi)\right. \\
& \left.+\left(\delta_{j k}-2 \hat{R}_{j} \hat{R}_{k}\right) \sin \Delta \psi \cos (l \Delta \psi)\right)+\left(\frac{\left(\delta_{j k}-3 \hat{R}_{j} \hat{R}_{k}\right) \sin \left(\sqrt{2} k r(1-\cos \Delta \psi)^{1 / 2}\right)}{2 k^{2} r^{2}(1-\cos \Delta \psi)^{2}}\right. \\
& \left.+\frac{\left(\delta_{j k}-3 \hat{R}_{j} \hat{R}_{k}\right) \cos \left(\sqrt{2} k r(1-\cos \Delta \psi)^{1 / 2}\right)}{2 \sqrt{2} k^{3} r^{3}(1-\cos \Delta \psi)^{5 / 2}}\right)\left(l \sin (l \Delta \psi)(1-\cos \Delta \psi)+\frac{3}{2} \sin \Delta \psi\right. \\
& \times \cos (l \Delta \psi))\}+3 \operatorname{Re}\left(\varepsilon_{i}^{(1)} \bar{\varepsilon}_{l}^{(2)}\left[\beta_{i j l}^{\mathrm{A}} \mu_{k}^{\mathrm{B}}+\beta_{i j l}^{\mathrm{B}} \mu_{k}^{\mathrm{A}}\right]\right) \frac{\left(\delta_{j k}-3 \hat{R}_{j} \hat{R}_{k}\right) \sin \Delta \psi}{\left.2 \sqrt{2} k^{3} r^{3}(1-\cos \Delta \psi)^{5 / 2}\right],}
\end{aligned}
$$

here and henceforth suppressing the implicit $f_{l p}$ and $\alpha_{i j}$ dependence.

\section{B. Spherical nanoparticles}

A system that it is of particular interest is where spherical nanoparticles are trapped in an annular intensity region as in fig. 3. With an irradiating LG beam, the laser-induced energy shift for the system is determined by expressing the polarization unit vector in simplified cylindrical coordinates. It is usually reasonable to exploit the condition $\mu_{k}^{\xi}=0$ for 
spherical particles (unless they are specially fabricated, when the dipolar terms can be retained), and hence equation (7) yields;

$$
\begin{aligned}
\Delta E_{\text {ind }}= & \left(\frac{I f_{l p}^{2}}{\varepsilon_{0} c A_{l p}}\right) \operatorname{Re}\left\{\sum_{J, K} \sin ^{2} \phi \cdot \alpha_{Z J}^{\mathrm{A}} V_{J K} \alpha_{K Z}^{\mathrm{B}}-\sin \phi \cos \phi \cdot \alpha_{Z J}^{\mathrm{A}} V_{J K} \alpha_{K X}^{\mathrm{B}}\right. \\
& \left.-\sin \phi \cos \phi \cdot \alpha_{X J}^{\mathrm{A}} V_{J K} \alpha_{K Z}^{\mathrm{B}}+\cos ^{2} \phi \cdot \alpha_{X J}^{\mathrm{A}} V_{J K} \alpha_{K X}^{\mathrm{B}}\right\} \cos (l \Delta \psi) .
\end{aligned}
$$

Taking the explicit distance and orientation-dependent form of the $V_{J K}$ tensor, and recognizing that only $\alpha_{\mathrm{XX}}=\alpha_{\mathrm{ZZ}}=\alpha$ exist for a spherical pair, equation (10) is rewritten as;

$$
\begin{aligned}
\Delta E_{\text {ind }}= & \left(\frac{I f_{l p}^{2} \alpha^{2}}{4 \pi \varepsilon_{0}^{2} c A_{l p}}\right)\left\{\cos ^{2} \phi\left(\frac{\cos k R}{R^{3}}+\frac{k \sin k R}{R^{2}}-\frac{k^{2} \cos k R}{R}\right)\right. \\
& \left.-2 \sin ^{2} \phi\left(\frac{\cos k R}{R^{3}}+\frac{k \sin k R}{R^{2}}\right)\right\} \cos (l \Delta \psi) .
\end{aligned}
$$

In this case the laser-induced torque results in the following expression;

$$
\begin{aligned}
\tau & =\left(\frac{I k^{3} f_{l p}^{2} \alpha^{2} \hat{\mathbf{R}}}{4 \pi \varepsilon_{0}^{2} c A_{l p}}\right)\left\{-\frac{\sin \left(\sqrt{2} k r(1-\cos \Delta \psi)^{1 / 2}\right)}{2(1-\cos \Delta \psi)} \cos ^{2} \phi \sin \Delta \psi \cos (l \Delta \psi)-\frac{\cos \left(\sqrt{2} k r(1-\cos \Delta \psi)^{1 / 2}\right)}{2 \sqrt{2} k r(1-\cos \Delta \psi)^{3 / 2}}\right. \\
& \times\left(2 l \cos ^{2} \phi \sin (l \Delta \psi)(1-\cos \Delta \psi)+\left(\left(1-3 \sin ^{2} \psi\right)+\cos ^{2} \psi\right) \sin \Delta \psi \cos (l \Delta \psi)\right)\left(\left(1-3 \sin ^{2} \psi\right)\right. \\
& \left.\times \frac{\sin \left(\sqrt{2} k r(1-\cos \Delta \psi)^{1 / 2}\right)}{2 k^{2} r^{2}(1-\cos \Delta \psi)^{2}}+\frac{\left(1-3 \sin ^{2} \phi\right) \cos \left(\sqrt{2} k r(1-\cos \Delta \psi)^{1 / 2}\right)}{2 \sqrt{2} k^{3} r^{3}(1-\cos \Delta \psi)^{5 / 2}}\right)(l \sin (l \Delta \psi)(1-\cos \Delta \psi) \\
& \left.\left.+\frac{3}{2} \sin \Delta \psi \cos (l \Delta \psi)\right)\right\} .
\end{aligned}
$$

In the short-range region $(k r \ll 1)$, signifying the usual and entirely appropriate condition that the nanoparticles are separated by a distance much less than the wavelength of the throughput radiation, equation (12) becomes;

$$
\begin{aligned}
\tau^{0}= & \frac{I f_{l p}^{2} \alpha^{2}\left(1-3 \sin ^{2} \phi\right) \cos \left(\sqrt{2} k r(1-\cos \Delta \psi)^{1 / 2}\right) \hat{\mathbf{R}}}{8 \sqrt{2} \pi \varepsilon_{0}^{2} r^{3} c A_{l p}(1-\cos \Delta \psi)^{5 / 2}}(l \sin (l \Delta \psi)(1-\cos \Delta \psi) \\
& \left.\left.+\frac{3}{2} \sin \Delta \varphi \cos (l \Delta \psi)\right)\right]
\end{aligned}
$$

On inspection of (12) and (13) it is apparent that, in comparison to a pair of nanoparticles irradiated by conventional Gaussian laser light $(l=0$, for example) additional contributions to the inter-particle torque will be observed with an LG beam $(l \neq 0)$. In the short-range region, and for three different values of $l$, graphs detailing the change in magnitude of $\tau^{0}$ with $\Delta \psi$ are exhibited in figs $4(\mathrm{a})-(\mathrm{c})$. Although the rise of $\tau^{0}$ towards infinity as $\Delta \psi$ approaches $0^{\circ}\left(360^{\circ}\right)$ is a prominent feature on these diagrams, it is an artifice corresponding to the non-physical case of particle overlap. Of greater interest is the physically significant observation that there are $(2 l-1)$ nodes, i.e. angular positions where $\tau^{0}=0$, of which it may be ascertained that $(l-1)$ correspond to maxima and $l$ to minima in $\Delta E_{\text {ind }}$. The latter energy minima signify stable values of $\Delta \psi$ for which the nanoparticle pair is mutually trapped. The above findings illustrate that the 
laser-induced mechanisms operative here differ very significantly from those of established 'optical spanner' methods not least because, here, there is no involvement of any orbital angular momentum transfer from the laser beam. It is the beam structure that engenders the potential energy distribution. Further distinctions can be identified in comparison to dynamic holographic optical tweezers schemes ${ }^{19}$ including the fact that here, the trapped particles do not circulate around the annular high intensity region of the optical vortex.

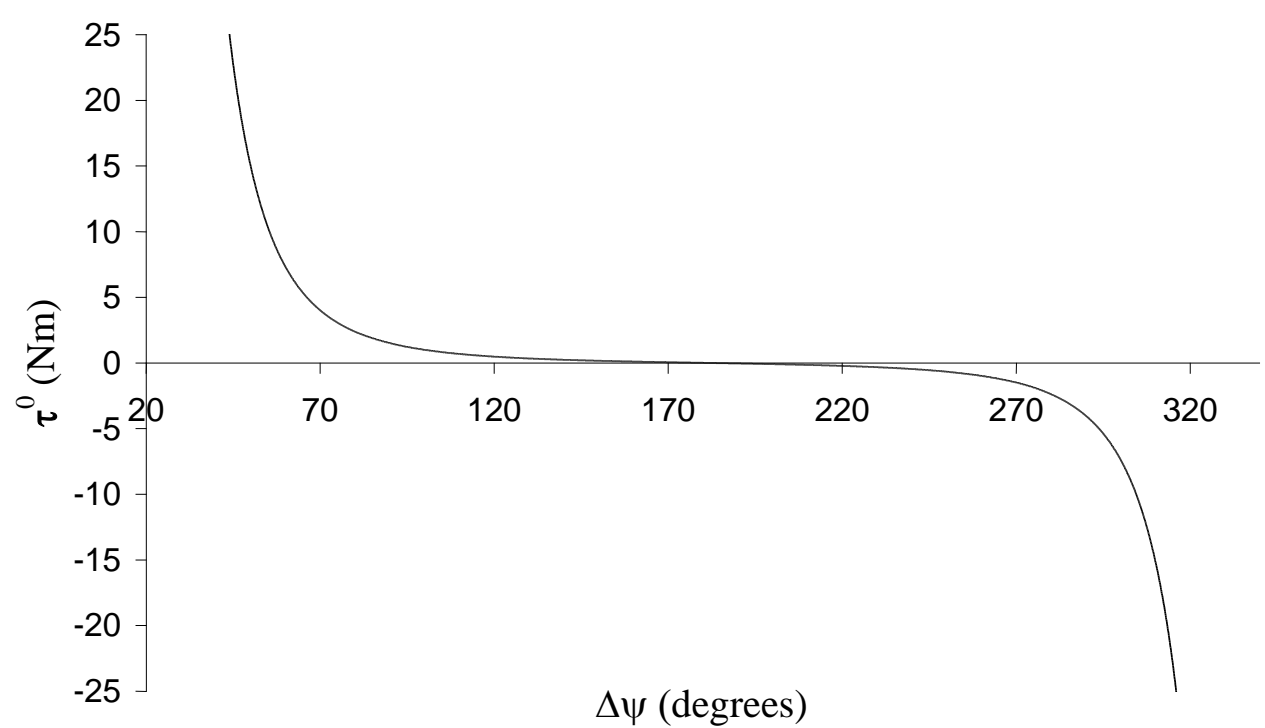

(a)

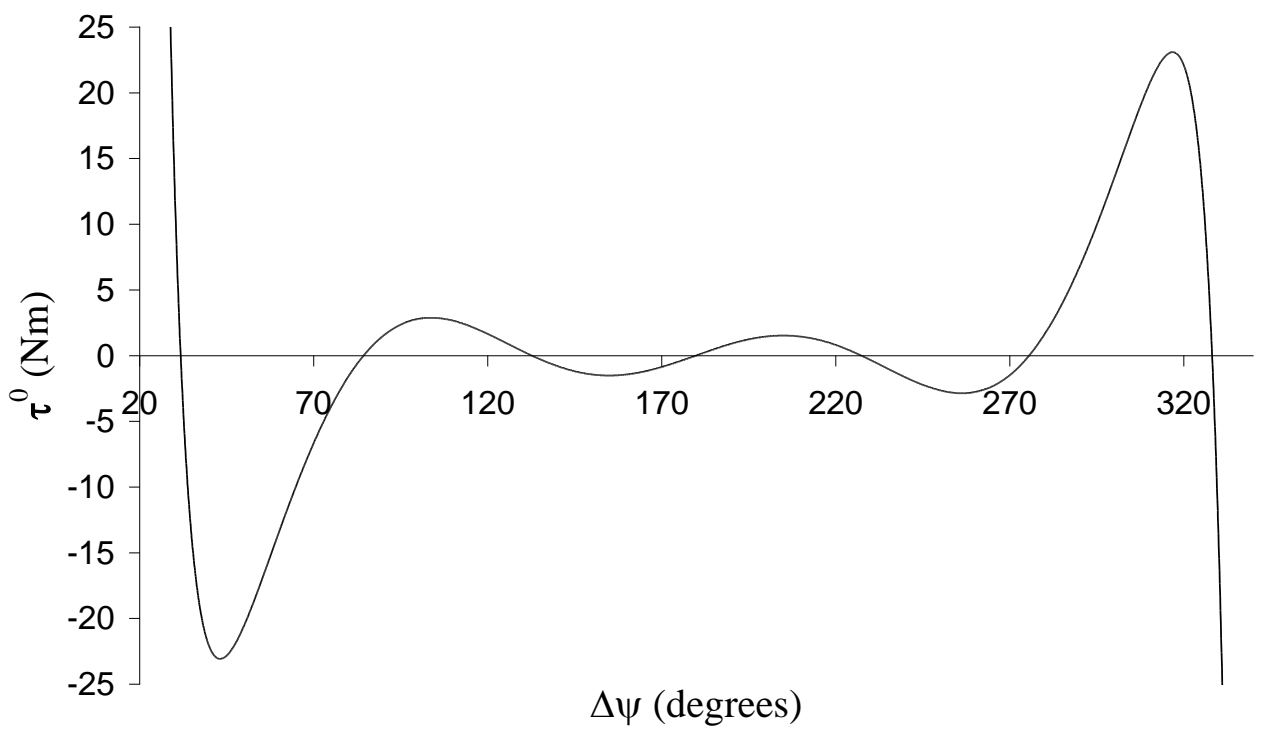

(b)

Fig. 4. Graphs of the magnitude of $\tau^{0}$ plotted against $\Delta \psi$ for a nanoparticle pair in a LG beam with: (a) $l=0$; (b) $l=4$. 


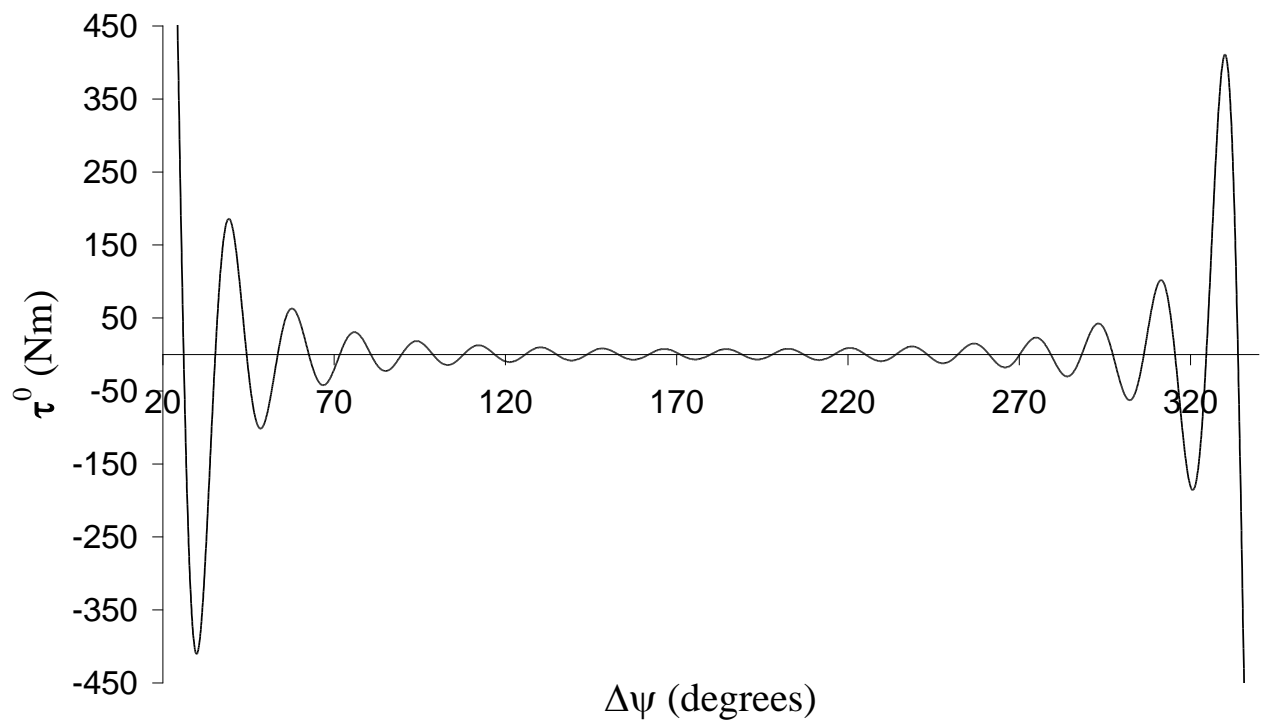

(c)

Fig. 4 (c). Graph of $\tau^{0}$ against $\Delta \psi$ for a nanoparticle pair in a LG beam with $l=20$.

\section{DISCUSSION}

It has been shown possible to significantly modify the form and magnitude of inter-particle forces and torques by the throughput of intense laser light. When optically twisted beams are involved, additional and highly distinctive torques have been shown to operate between pairs of nanoparticles; such forces have the capacity to generate novel patterns of nanoscale response, fully determined by the frequency, intensity, polarization and other experimentally controllable properties of the laser input. It is interesting to discover that these laser-induced forces and torques can be either positive or negative according to conditions, in contrast to 'optical binding'. As a consequence, new possibilities emerge for the optical control of nanoparticle ordering, clustering and trapping.

\section{APPENDIX}

State-sequence diagrams ${ }^{20}$ are a relatively new graphic representation in which quantum electrodynamical interactions are easily visualized, and which also greatly assist in calculations. Examples of these diagrams are given in figs 1 and 2. Each box in the state-sequence diagram is linked to a reference position $(m, k)$, where $m$ and $k$ are the vertex and step number, respectively, and a matter-photon interaction event is denoted by a connector between boxes. Therefore, for example, the lowest quantum channel is represented by $|i\rangle \rightarrow\left|r_{1}^{1}\right\rangle \rightarrow\left|r_{2}^{1}\right\rangle \rightarrow\left|r_{3}^{1}\right\rangle \rightarrow|f\rangle$, where $|i\rangle,|f\rangle$ and $\left|r_{k}^{m}\right\rangle$ are the initial, final and intermediate states, respectively. In each state-sequence diagram (e.g. either fig. 1 or 2) twenty-four quantum channels can be traced from the initial to the final state, each corresponding to one member of a complete set of contributions to the quantum amplitude. To perform the ensuing calculations using the more familiar Feynman (time-ordered) representation, the construction of twenty-four diagrams would be required. There are several further advantages over the more familiar time-ordered diagrams including: (i) the connectivity of different quantum channels is readily identifiable; (ii) salient parameters for states that feature in several channels need only be computed once; (iii) all time-orderings for a particular process are embedded in a single graph; (iv) results associated with higher order corrections are calculable from the same graph without modification.

\section{ACKNOWLEDGMENT}

DSB is funded by a studentship from the Engineering and Physical Sciences Research Council. 


\section{REFERENCES}

1. A. Ashkin, “Optical trapping and manipulation of neutral particles using lasers”, Proc. Natl. Acad. Sci. USA 94, pp. 4853-4860, 1997.

2. J. E. Molloy and M. J. Padgett, "Light, action: optical tweezers", Contemp. Phys. 43, pp. 241-258, 2002.

3. K. Svoboda and S. M. Block, "Biological applications of optical forces", Annu. Rev. Biophys. Biomol. Struct. 23, pp. 247-285, 1994.

4. V. I. Balykin, V. G. Minogin and V. S. Letokhov, "Electromagnetic trapping of cold atoms", Rep. Prog. Phys. 63, pp. 1429-1510, 2000.

5. T. A. Nieminen, H. Rubinsztein-Dunlop and N. R. Heckenberg, "Calculation and optical measurement of laser trapping forces on non-spherical particles”, J. Quant. Spectrosc. Rad. Trans. 70, pp. 627-637 (2001).

6. D. G. Grier, “A revolution in optical manipulation”, Nature 424, pp. 810-816, 2003.

7. N. B. Simpson, K. Dholakia, L. Allen and M. J. Padgett, "Mechanical equivalence of spin and orbital angular momentum of light: an optical spanner", Opt. Lett. 22, pp. 52-54, 1997.

8. T. A. Nieminen, N. R. Heckenberg and H. Rubinsztein-Dunlop, "Optical measurement of microscopic torque”, J. Mod. Opt. 48, pp. 405-413, 2001.

9. T. Thirunamachandran, "Intermolecular interactions in the presence of an intense radiation field", Mol. Phys. 40, pp. 393-399, 1980.

10. D. L. Andrews and D. S. Bradshaw, "Laser-induced forces between carbon nanotubes”, Opt. Lett. 30, pp. 783-785, 2005.

11. L. C. Dávila Romero, D. L. Andrews and M. Babiker, "A quantum electrodynamics framework for the nonlinear optics of twisted beams”, J. Opt. B: Quantum Semiclass. Opt. 4, pp. S66-S72, 2002.

12. D. P. Craig and T. Thirunamachandran, Molecular Quantum Electrodynamics (Dover, Mineola, New York, 1998).

13. R. G. Woolley, "Gauge invariance in non-relativistic electrodynamics”, Proc. R. Soc. Lond. A 456, pp. 1803-1819, 2000 .

14. D. S. Bradshaw and D. L. Andrews, "Optical forces between dielectric nanoparticles in an optical vortex", in Nanomanipulation with Light, ed. D. L. Andrews, Proc. SPIE 5736, 87-95, 2005.

15. G. J. Daniels, R. D. Jenkins, D. S. Bradshaw and D. L. Andrews, "Resonance energy transfer: The unified theory revisited", J. Chem. Phys. 119, pp. 2264-2274, 2003.

16. F. Depasse and J.-M. Vigoureux, “Optical binding force between two Rayleigh particles”, J. Phys. D: Appl. Phys. 27, pp. 914-919, 1994.

17. L. Allen, M. J. Padgett and M. Babiker, "The orbital angular momentum of light”, Progr. Opt. 39, pp. 291-372, 1999.

18. L. Allen, S. M. Barnett and M. J. Padgett, Optical Angular Momentum (IOP, Bristol, 2003).

19. J. E. Curtis, B. A. Koss and D. G. Grier, "Dynamic holographic tweezers", Opt. Comm. 207, pp. 169-175, 2002.

20. R. D. Jenkins, D. L. Andrews and L. C. Dávila Romero, "A new diagrammatic methodology for non-relativistic quantum electrodynamics”, J. Phys. B: At. Mol. Opt. Phys. 35, pp. 445-468, 2002.

*david.andrews@physics.org 